\title{
ROLE OF ULTRASOUND STRAIN ELASTOGRAPHY FOR THE EVALUATION OF BREAST LESIONS
}

\begin{tabular}{ll}
$\begin{array}{l}\text { Dr. Deoyani S } \\
\text { Sarjare* }\end{array}$ & $\begin{array}{l}\text { Government Medical College, Nagpur, Maharashtra. }{ }^{*} \text { Corresponding } \\
\text { Author }\end{array}$ \\
\hline $\begin{array}{l}\text { Dr. Arti Anand } \\
\begin{array}{l}\text { Dr. Soumya } \\
\text { Agrawal }\end{array}\end{array}$ & Government Medical College, Nagpur, Maharashtra. \\
\hline $\begin{array}{l}\text { Dr. Shanas K.P. } \\
\begin{array}{l}\text { Dr. Sandhya } \\
\text { Yeshwante }\end{array}\end{array}$ & Government Medical College, Nagpur, Maharashtra. \\
\hline \hline
\end{tabular}

ABSTRACT Introduction: Ultrasound (US) elastography is an emerging technique that can be used during breast US examination. Guidelines recommend mammographic screening and US for diagnosis of breast cancer. The specificity of these techniques is not high enough to prevent unnecessary biopsies. Hence there is need for a more specific technique to overcome this problem. This study aimed to evaluate the value of strain elastography (SE) for breast lesions. Materials And Methods: In this cross sectional observational study over 18 months, 60 women with palpable breast lumps were evaluated with conventional US and SE. Results obtained were correlated with histopathological findings for statistical analysis. Result: $\mathrm{A}$ statistically significant correlation was found between SE and histopathological outcome with a p value of $0.03(<0.05)$. The positive predictive value (PPV) was $76.92 \%$ and the negative predictive value (NPV) was $76.47 \%$ with Chi square= 8.4. Conclusion: Ultrasound with SE can substantially improve the possibility of differentiating benign from malignant breast lesions thereby limiting recourse to biopsy and considerably reducing the number of benign breast biopsy diagnoses.

\section{KEYWORDS : elastography; strain imaging; sonomammography; breast lesions}

INTRODUCTION

The high incidence of breast cancer and its slow evolution before diagnosis led to research on new diagnostic techniques. Mammography and US have highest sensitivity for diagnosis of breast cancer. However, mammography performed in dense breasts often yields false-negative results [1]. US has low specificity as most solid lesions are benign. To increase the specificity, breast lesions are classified into ACR BI-RADS category ${ }^{[2]}$. This increases the number of false positive results and suspicious lesions that are subjected to biopsies with low cancer "detection rate" of only $10 \%-30 \%{ }^{[3,4,5]}$.

To overcome these limitations, US elastography was introduced. Breast elastography is a novel, non invasive sonographic imaging technique which uses the differences in the specific elastic modulus of tissues. It measures the deformation of tissues which can be detected by applying an external mechanical force ${ }^{[6,7]}$. Tissue deformation is inversely proportional to its stiffness ${ }^{[8]}$. Benign lesions have elasticity similar to the surrounding tissue and are deformable, while malignant lesions are harder and appear larger than adjacent tissue owing to intra and extranodular desmoplastic reaction or by neoplastic infiltration of the interstitial tissue ${ }^{[4]}$. The ratio between the diameter of the lesion on the elastogram as compared to the US image; a ratio of $\geq 1$ is suggestive of malignancy ${ }^{[9,10]}$.

Elastographic scoring system suggested by Itoh et al. ${ }^{[11]}$ is called as TSUKUBA score which assigns a score from 1 to $\overline{5}$ as follows: Score 1: entire lesion is deformable, Score 2: most of the lesion is deformable with some small stiff areas (Fig 1),
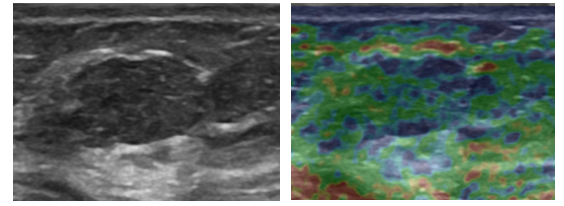

Figl. Conventional USG and elastography image showing well defined BiRADS 2 lesion with Tsukuba score 2 on elastography, s/o benign nature.
Score 3: peripheral portion of the lesion is deformable with stiff tissue in the center (Fig 2)
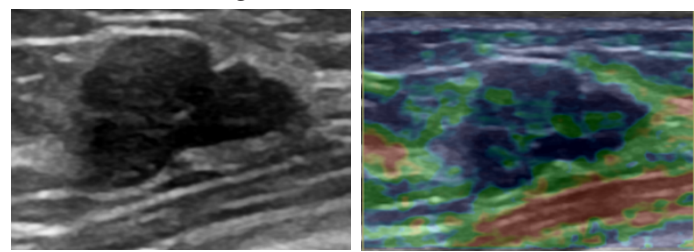

Fig 2. Conventional USG and elastography image showing BIRADS 3 with Tsukuba score 3 on elastography.

Score 4: entire lesion is stiff, Score 5: the entire lesion and surrounding tissue are stiff (Fig.3).
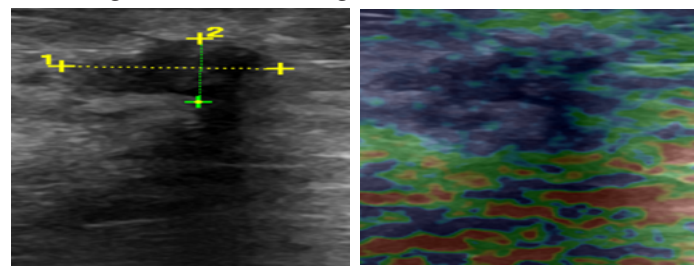

Fig 3. Conventional USG and elastography image showing BIRADS 4 lesion with Tsukuba score 5 s/o malignant lesion.

Score 1 to 3 suggests benign whereas score 4 and 5 suggests malignant lesion. Simple cysts are not deformable but produce a typical "tri-stratified" or "target" pattern of artifact [12]. (Fig 4)
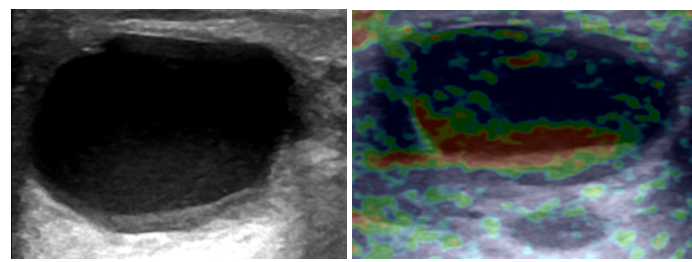

Fig 4. Conventional USG and elastography image showing 
simple cyst with BGR (blue -green-red) layering artefacts on elastography.

SE has several specific applications- BI-RADS category $4 a$ masses with soft elasticity can be downgraded to category 3 , reducing unnecessary biopsies.

BI-RADS category 3 lesions with very soft elasticity can safely be downgraded to category 2 , thereby reducing unnecessary short-term follow-up.

Benign appearing well circumscribed malignant lesions that are misclassified as BI-RADS category 3 on B-mode US can be accurately diagnosed as malignant owing to their stiffness on SE (Fig 5).

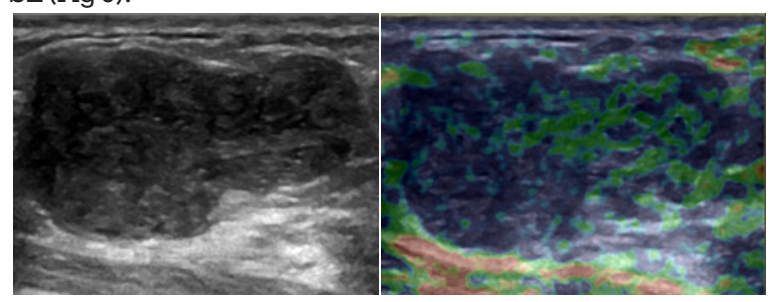

Fig 5. Conventional USG and elastography image showing BIRADS 3 lesion with Tsukuba score 5 on elastography, thus upgrading it to BIRADS 4a.

In addition, fat lobules or benign cysts, which can mimic solid breast masses, can be identified by their markedly soft elasticity thereby increasing the level of diagnostic confidence.

There are 2 different types of techniques widely used for determination of the rigidity of the lesion with US; strain elastography and shear wave elastography techniques. In strain elastography, the observer or the patient produces the pressure either with the probe or by breathing whereas in the shear wave technique that pressure is formed by the US probe by a special sound wave, which is called shear wave ${ }^{[13] .}$

The most widely used method currently is real-time elastography (RTE) which generates "strain imaging" by compression and is performed using conventional US equipment with dedicated software. It evaluates the relative elasticity of the tissues in a specific area of interest (the RTEbox) creating a colour coded map called as elastogram that is superimposed to the US image and updated in real-time at a frequency of $10-15 \mathrm{~Hz}{ }^{[14]}$. The areas of great stiffness are coded in blue, those which are more deformable in red, and green indicates intermediate levels of elasticity.

The purpose of this study was to assess the role of SE in the diagnosis of breast lesions.

\section{Aims And Objectives}

1. To differentiate breast masses into benign and malignant category US and SE.

2. Correlation of the imaging findings with histopathological results.

\section{Materials And Methods:}

This cross sectional observational study was conducted over 60 female patients in the age group of 21 to 78 years who presented with palpable breast lumps and were referred from the Department of Obstetrics and Gynaecology over a period of 18 months from August 2018 to Feb 2020.

All female patients referred for sonomammography for palpable lumps. Patients with new mass lesion in a previously irradiated breast were excluded from the study.

All the patients underwent B mode US evaluation followed by elastography on GE LOGIQ S8 machine with high frequency small part linear probe $(5-18 \mathrm{~Hz})$ and lesions were classified according to BI-RADS. US elastography was performed while the patient was in supine position and the transducer was placed vertical to the lesion. Compression was applied with the probe over the lesion and elastographic images were examined. The elasticity region of interest (ROI) was placed to cover the lesion and the target lesion was placed in the center. The elastogram was superimposed on B mode ultrasound image and was displayed besides the US image by a split screen function. Elastography was followed by FNAC/ biopsy correlation. The results were tabulated for statistical analysis.

\section{RESULTS:}

Sixty female patients in the age group from 21 to 78 years were evaluated with conventional US and SE. Thirty three percent patients were in the age group of more than 60 years, $26 \%$ in the age group of $41-50$ years and $6.6 \%$ in less than 30 years age group. The mean age of affection was 52.40 years. Twelve cases $(20 \%)$ had family history of breast cancer.

Most of the lesions (60\%) were hypoechoic. 10 lesions had mixed echogenicity (33.3\%), whereas 4 lesions were anechoic $(6.66 \%)$.Twenty four lesions were wider than taller $(40 \%)$, whereas 36 lesions were taller than wider (60\%).Thirty six lesions had lobulated margins (60\%), 22 had irregular margins (36.66\%), whereas 4 lesions had smooth margin (3.33 $\%)$.Intralesional calcification and vascularity were present in $40(66.6 \%)$ and 50 lesions respectively. Maximum no of lesions (66.6\%) were BIRADS IV (likelihood of cancer being 2-95\%). $26.6 \%$ lesions were BIRADS V (likelihood of cancer $\geq 95$ ).

Twenty eight lesions had elastography score of 4 , (46.6\%). 22 patients had score of 1 , (36.66\%).Twenty six lesions (43.3\%) were benign whereas 34 lesions $(56.66 \%)$ were suggestive of malignant nature on SE (Tablel).

32 malignant lesions (53\%) and 28 (46.6\%) benign lesions were found on histopathological evaluation (Table 2).

Tablel. Classification Of Lesions Based On Elastography Score

\begin{tabular}{|l|l|l|}
\hline Elastography score & No. of lesions & Percentage (\%) \\
\hline 1 & 22 & 36.66 \\
\hline 2 & 2 & 3.33 \\
\hline 3 & 2 & 3.33 \\
\hline 4 & 28 & 46.66 \\
\hline 5 & 6 & 10.00 \\
\hline Total & 60 & 100 \\
\hline
\end{tabular}

Of the histopathologically confirmed 32malignant lesions, 26 were correctly identified as malignant by US and SE each. However, 24/28 benign lesions were correctly diagnosed on US and 26/ 28 lesions were correctly diagnosed on SE(Table 2 and Table 3).

Table 2. Correlation Between US And Histopathology Findings

\begin{tabular}{|l|l|l|l|l|l|l|}
\hline \multirow{2}{*}{$\begin{array}{l}\text { USG } \\
\text { finding }\end{array}$} & \multicolumn{4}{|l|}{ HPE } & Total & P \\
\cline { 2 - 5 } & Benign & \multicolumn{3}{|l|}{ Malignant } & Value \\
\cline { 2 - 5 } & Frequency & $\%$ & Frequency & $\%$ & & \\
\hline Benign & 18 & $64.2 \%$ & 6 & $18.75 \%$ & 24 & .01 \\
\hline Malignant & 10 & $35.71 \%$ & 26 & $81.25 \%$ & 36 & \\
\hline Total & 28 & $100 \%$ & 32 & $100 \%$ & 60 & \\
\hline
\end{tabular}

Table 3. Correlation Between Elastography And Histopathology Findings

\begin{tabular}{|l|l|l|l|l|l|l|}
\hline Elastography & HPE & Total & P \\
\cline { 2 - 5 } & Benign & \multicolumn{2}{|l|}{ Malignant } & Value \\
\cline { 2 - 5 } & $\begin{array}{l}\text { No. of } \\
\text { lesions }\end{array}$ & $\begin{array}{l}\text { No. of } \\
\text { lesions }\end{array}$ & $\%$ & & \\
\hline Benign & 20 & 71.42 & 6 & 28.50 & 26 & 0.03 \\
\hline
\end{tabular}




\begin{tabular}{|l|l|l|l|l|l|l|}
\hline Malignant & 8 & 28.50 & 26 & 81.25 & 34 & \\
\hline Total & 28 & $100 \%$ & 32 & $100 \%$ & 60 & \\
\hline
\end{tabular}

\section{DISCUSSION}

In our study, 18/28 benign lesions and 26/32 malignant lesions were diagnosed on US with sensitivity $81 \%$ but low specificity of $64 \%$, PPV was $72 \%$ and NPV was $75.22 \%$. A statistically significant correlation was found between the conventional ultrasound findings and histopathological examination with a $p$ value $<0.01$ with Chi square $=6.4509$ and high accuracy of the test $(73.33 \%)$

In our study, 20/28 benign lesions and 26/32 malignant lesions were diagnosed on SE with sensitivity $81 \%$, higher specificity $71 \%$, PPV was $76.47 \%$ and NPV was $76.92 \%$. Statistically significant correlation was found between the SE with histopathological outcome, p value of $0.03(<0.05)$ and Chi square $=8.4$

We found $8 / 34$ false positive malignant lesions and 6/ 26 false negative malignant lesions. Most of our lesions had diameter more than $20 \mathrm{~mm}$ with very few lesions smaller than $5 \mathrm{~mm}$. Giuseppetti et al. [15] reported a better diagnostic performance in lesions smaller than $2 \mathrm{~cm}$. Scaperrotta et al [16] did not find statistically significant differences in the diagnostic performance between small and large lesions. A recent meta-analysis of Sadigj $G$ et al. [17] showed that regardless of the lesion size, US elastography had a higher specificity and lower sensitivity as compared to B mode US in characterizing breast masses.

There were 2 benign lesions with elastography scores of 2 , but having malignant features and so were classified as BI-RADS category $4 \mathrm{~b}$ according to $B$ mode US. We believe that lesions evaluated as score 2 can be accepted as benign, unless they present suspicious malignant features on B Mode US. Our findings are comparable with the recent literature that biopsies of BI-RADS 3 and 4 a lesions can be prevented with the aid of strain elastography [18].

Our study had few limitations. The effects of breast density and composure on elastography results were not evaluated in our study. SE is operator dependent and subject to intraobserver or interobserver variability.

\section{Summary}

Role of SE for differentiating benign from malignant breast masses was evaluated. US and SE was performed on 60 females with palpable lumps and results were compared with histopathological reports. Statistically significant correlation was found between US and histopathology as well as SE and histopathology. However, SE showed higher specificity than US for diagnosis of malignant breast lesions. Few limitations were observed in the study due to larger size of lesions and interobserver variability, inaccurate techniques of performing US with SE.

\section{CONCLUSION}

US with SE may reduce the need for biopsy in soft benign lesions classified as BI-RADS 3 on US image and postpone follow-up. It has a significant role in upgrading benign looking hard malignant lesions, thereby resulting in early diagnosis of malignant lesions. Elastography has a significant role in the management of nodules $<5 \mathrm{~mm}$ which are visible on the US image, but not on mammography, in which reduced deformability may lead to biopsy rather than monitoring as required by the current guidelines. Elastography can aid in increasing the level of diagnostic confidence with respect to lesions containing fat, fluid.

$\mathrm{SE}$ is useful in the assessment of elastic tissue properties thanks to the short examination time required, real-time display, immediate interpretation and limited cost, and the clinically adequate criteria adopted for the image interpretation. We recommend that all patients must undergo elastographic imaging along with US for comprehensive tissue imaging of breast masses which can aid to plan the further diagnostic and management strategy for each breast lesion. We also recommended that aqeduate training and knowledge must be inculcated amongst the radiologists for optimum utilization of $\mathrm{SE}$ and help in better patient outcome.

\section{REFERENCES:}

1. Saarenmaa I., Salminen T., Geiger U., Heikkinen P., Hyvärinen S., Isola J. The effect of age and density of the breast on the sensitivity of breast cancer diagnostic by mammography and ultrasonography. Breast Cancer Res Treat. 2001;67:117-123. [PubMed] [Google Scholar]

2. Breast imaging reporting and data system (BI-RADS). http://www.acr.org

3. Athanasiou A., Tardivon A., Tanter M., Sigal-Zafrani B., Bercoff J., Deffieux T Breast lesions: quantitative elastography with supersonic shear imaging preliminary results. Radiology. 2010;256:297-303. [PubMed] [Google Scholar]

4. Duncan J.L., 3rd, Cederbom G.J., Champaign J.L., Smetherman D.H., King T.A., Farr G.H. Benign diagnosis by image-guided core-needle breast biopsy. Am Surg. 2000 Jan;66(1):5-9. discussion 9-10. [PubMed] [Google Scholar]

5. Chiou S.Y., Chou Y.H., Chiou H.J., Wang H.K., Tiu C.M., Tseng L.M. Sonographic features of nonpalpable breast cancer: a study based on ultrasound-guided wire-localized surgical biopsies. Ultrasound Med Biol. 2006:32:1299-1306. [PubMed] [Google Scholar]

6. Chen E.J., Novakofski J., Jenkins W.K., O'Brien W.D. Young's modulus measurements of soft tissues with application to elasticity imaging. IEEE Trans Ultrason Ferroelectr Freq Control. 1996;43:191-194. [Google Scholar]

7. Frey H. Realtime elastography. A new ultrasound procedure for the reconstruction of tissue elasticity. Radiologe. 2003;43:850-855. [PubMed] [Google Scholar]

8. Nightingale K., Soo M.S., Nightingale R., Bentley R., Trahey G. In vivo demonstration of acoustic radiation force impulse (ARFI) imaging in the thyroid, abdomen, and breast. Ultrasonics symposium. IEEE. 2001;2:1633 1638. [Google Scholar]

9. Barr R.G. Radiological society of North America scientific assembly and annual meeting program. RSNA; Oak Brook, IL: 2006. Initial results of breast real-time elasticity imaging to characterize lesions [abstr] p. 644. [Google Scholar]

10. Barr R.G., Destounis S., Lackey L.B., 2nd, Svensson W.E., Balleyguier C. Smith C. Evaluation of breast lesions using sonographic elasticity imaging: $a$ multicenter trial. J Ultrasound Med. 2012;31:281-287. [PubMed] [Google Scholar]

10. Nightingale K., Bentley R., Trahey G. Observations of tissue response to acoustic radiation force: opportunities for imaging. Ultrason Imaging 2002:24:129-138. [PubMed] [Google Scholar]

11. Itoh A., Ueno E., Tohno E., Kamma H., Takahashi H., Shiina T. Breast disease: clinical application of US elastography for diagnosis. Radiology. 2006; 239: 341-350. [PubMed] [Google Scholar]

12. Barr R.G., Lackey A.E. The utility of the "bull's-eye" artifact on breast elasticity imaging in reducing breast lesion biopsy rate. Ultrasound Q. 2011;27: 151155. [PubMed] [Google Scholar]

13. Gennisson JL, Deffieux T, Fink M, Tanter M. Ultrasound elastography Principles and technique. Diagnostic and Interventional Imaging: 94; 2013 487-495. (PMID: 23619292) [

14. Shiina T., Nitta N., Ueno E., Bamber J.C. Real time tissue elasticity imaging using the combined autocorrelation method. I Med Ultrason. 2002.29: 119 128. [PubMed] [Google Scholar] 25. Nightingale K., Stutz D., Bentley R. Trahey G. Acoustic radiation force impulse imaging: ex vivo and in vivo demonstration of transient shear wave propagation. Biomed Imag. 2002:525-528. Conference Publications. [Google Scholar]

15. Giuseppetti GM, Martegani A, Di Cioccio B, Baldassarre S. Elastosono graphy in the diagnosis of the nodular breast lesions: preliminary report. Radiol Med 2005; 110:69-76. (PMID: 16163141) 7

16. Scaperrotta G, Ferranti C, Costa C, Mariani L, Marchesini M, Suman L, Folin C, Bergonzi S. Role of sonoelastography in non-palpable breast lesions. Eur Radiol 2008; 18:2381-2389. (PMID: 18523780)

17. Sadigh G, Carlos RC, Neal CH, Wojcinski S, Dwamena BA. Impact of breast mass size on accuracy of ultrasound elastography vs. conventional B-mode ultrasound: a meta-analysis of individual participants.

18. Cho N, Moon WK, Park JS, Cha JH, Jang M, Seong MH. Nonpalpable breast masses: evaluation by US elastography. Korean J Radiol 2008; 9:111-118. (PMID: 18385557) 